\title{
Divisibility of L-Polynomials for a Family of Artin-Schreier Curves
}

\author{
Gary McGuire 1 and Emrah Sercan Yilmaz 2 \\ School of Mathematics and Statistics \\ University College Dublin \\ Ireland
}

\begin{abstract}
In this paper we consider the curves $C_{k}^{(p, a)}: y^{p}-y=x^{p^{k}+1}+a x$ defined over $\mathbb{F}_{p}$ and give a positive answer to a conjecture about a divisibility condition on $L$-polynomials of the curves $C_{k}^{(p, a)}$. Our proof involves finding an exact formula for the number of $\mathbb{F}_{p^{n}}$-rational points on $C_{k}^{(p, a)}$ for all $n$, and uses a result we proved elsewhere about the number of rational points on supersingular curves.
\end{abstract}

\section{Introduction}

Let $p$ be a prime and let $q=p^{r}$ where $r$ is a positive integer. Let $\mathbb{F}_{q}$ be the finite field with $q$ elements. Let $X$ be a projective smooth absolutely irreducible curve of genus $g$ defined over $\mathbb{F}_{q}$. The $L$-polynomial of the curve $X$ over $\mathbb{F}_{q}$ is defined by

$$
L_{X / \mathbb{F}_{q}}(T)=L_{X}(T)=\exp \left(\sum_{n=1}^{\infty}\left(\# X\left(\mathbb{F}_{q^{n}}\right)-q^{n}-1\right) \frac{T^{n}}{n}\right) .
$$

where $\# X\left(\mathbb{F}_{q^{n}}\right)$ denotes the number of $\mathbb{F}_{q^{n}}$-rational points of $X$. It is well known that $L_{X}(T)$ is a polynomial of degree $2 g$ with integer coefficients, so we write it as

$$
L_{X}(T)=\sum_{i=0}^{2 g} c_{i} T^{i}, c_{i} \in \mathbb{Z}
$$

It is also well known that $c_{0}=1$ and $c_{2 g}=q^{g}$.

\footnotetext{
${ }^{1}$ email gary.mcguire@ucd.ie, Research supported by Science Foundation Ireland Grant 13/IA/1914

${ }^{2}$ Research supported by Science Foundation Ireland Grant 13/IA/1914
} 
We wish to consider the question of divisibility of L-polynomials. In previous papers [2], [3], we have studied conditions on the curves under which the L-polynomial of one curve divides the L-polynomial of another curve. A theorem of Tate gives an answer in terms of Jacobians. We refer the reader to these papers for a longer discussion of this topic.

Artin-Schreier curves are degree $p$ coverings of the projective line, and are cyclic extensions of degree $p$ of the rational function field. It can be shown that all Artin-Schreier curves have an equation of the form $y^{p}-y=f(x)$. Let $k$ be a positive integer. In this paper we will study the family of Artin-Schreier curves

$$
C_{k}^{(p, a)}: y^{p}-y=x^{p^{k}+1}+a x
$$

where $a \in \mathbb{F}_{p}$, which are defined over $\mathbb{F}_{p}$ and have genus $p^{k}(p-1) / 2$. We will prove the following conjecture, which is stated in [4].

Conjecture 1. Let $k$ and $m$ be positive integers. Then the L-polynomial of $C_{k m}^{(p, a)}$ is divisible by the L-polynomial of $C_{k}^{(p, a)}$.

The L-polynomials in the conjecture are over $\mathbb{F}_{p}$. This conjecture was proved for $p=2$ in [4], so we will assume that $p$ is odd for this paper. In Section 10 we explain why we can assume $a=1$ without loss of generality. We prove this conjecture by finding an exact expression for

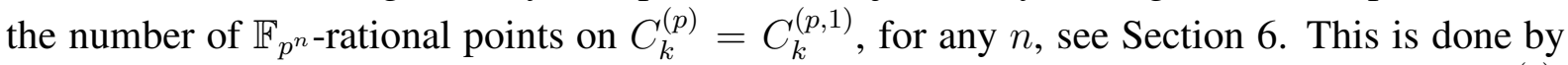
first finding an exact expression for the number of $\mathbb{F}_{p^{n}}$-rational points on related curves $B_{k}^{(p)}$, see Section 5. Sections 3 and 4 deal with the curves $B_{0}^{(p)}$ and $C_{0}^{(p)}$ respectively, which need separate consideration. Section 7 gives the proof of Conjecture 1 , In Section 8 we consider the corresponding divisibility result for the $B_{k}$ family. Section 9 contains some results on the opposite problem to the conjecture; we prove that if $k$ does not divide $\ell$ then the L-polynomial of $C_{k}^{(p)}$ does not divide the L-polynomial of $C_{\ell}^{(p)}$.

We will usually drop the superscript in $C_{k}^{(p)}=C_{k}^{(p, 1)}$, and write $C_{k}$. The trace map is always the absolute trace, unless otherwise stated. Throughout the paper $(\dot{\bar{p}})$ denotes the Legendre symbol. Finally, the L-polynomials of a more general class of curves than $C_{k}$ were found in [1], however they were L-polynomials over an extension of $\mathbb{F}_{p}$, and not L-polynomials over $\mathbb{F}_{p}$, which is the subject of this paper. 


\section{Background}

In this section we will give some basic facts that we will use. Some of this requires that $p$ is odd, some of it does not, but we remind that reader that we are going to assume $p$ is odd for this paper.

\subsection{More on Curves}

Let $p$ be a prime and let $q=p^{r}$ where $r$ is a positive integer. Let $X$ be a projective smooth absolutely irreducible curve of genus $g$ defined over $\mathbb{F}_{q}$. Let $\eta_{1}, \cdots, \eta_{2 g}$ be the roots of the reciprocal of the $L$-polynomial of $X$ over $\mathbb{F}_{q}$ (sometimes called the Weil numbers of $X$, or Frobenius eigenvalues). Then, for any $n \geq 1$, the number of rational points of $X$ over $\mathbb{F}_{q^{n}}$ is given by

$$
\# X\left(\mathbb{F}_{q^{n}}\right)=\left(q^{n}+1\right)-\sum_{i=1}^{2 g} \eta_{i}^{n} .
$$

The Riemann Hypothesis for curves over finite fields states that $\left|\eta_{i}\right|=\sqrt{q}$ for all $i=1, \ldots, 2 g$. It follows immediately from this property and (2) that

$$
\left|\# X\left(\mathbb{F}_{q^{n}}\right)-\left(q^{n}+1\right)\right| \leq 2 g \sqrt{q^{n}}
$$

which is the Hasse-Weil bound.

We call $X\left(\mathbb{F}_{q}\right)$ maximal if $\eta_{i}=-\sqrt{q}$ for all $i=1, \cdots, 2 g$, so the Hasse-Weil upper bound is met. Equivalently, $X\left(\mathbb{F}_{q}\right)$ is maximal if and only if $L_{X}(T)=(1+\sqrt{q} T)^{2 g}$.

We call $X\left(\mathbb{F}_{q}\right)$ minimal if $\eta_{i}=\sqrt{q}$ for all $i=1, \cdots, 2 g$, so the Hasse-Weil lower bound is met. Equivalently, $X\left(\mathbb{F}_{q}\right)$ is minimal if and only if $L_{X}(T)=(1-\sqrt{q} T)^{2 g}$.

Note that if $X\left(\mathbb{F}_{q}\right)$ is minimal or maximal then $q$ must be a square (i.e. $r$ must be even).

The following properties follow immediately.

Proposition 1. 1. If $X\left(\mathbb{F}_{q}\right)$ is maximal then $X\left(\mathbb{F}_{q^{n}}\right)$ is minimal for even $n$ and maximal for odd $n$.

2. If $X\left(\mathbb{F}_{q}\right)$ is minimal then $X\left(\mathbb{F}_{q^{n}}\right)$ is minimal for all $n$. 
We also record another Proposition here.

Proposition 2. If $X$ is a curve defined over $\mathbb{F}_{q}$ and $X\left(\mathbb{F}_{q^{2 n}}\right)$ is maximal, then $\# X\left(\mathbb{F}_{q^{n}}\right)=q^{n}+1$ and the L-polynomial of $X$ over $\mathbb{F}_{q^{n}}$ is $\left(1+q^{n} t^{2}\right)^{g}$.

Proof. Let $\eta_{1}, \cdots, \eta_{2 g}$ be the Weil numbers of $X$ over $\mathbb{F}_{q}$. Then $\eta_{j}^{2 n}=-\sqrt{q^{2 n}}=-q^{n}$ for all $j$ because $X\left(\mathbb{F}_{q^{2 n}}\right)$ is maximal. But then $\eta_{j}^{n}= \pm i \sqrt{q^{n}}$ for all $j$, which implies that

$$
\sum_{j=1}^{2 g} \eta_{j}^{n}=\left(q^{n}+1\right)-\# X\left(\mathbb{F}_{q^{n}}\right)
$$

is a purely imaginary complex number and also an integer. This number is therefore 0 .

\subsection{Supersingular Curves}

A curve $X$ of genus $g$ defined over $\mathbb{F}_{q}\left(q=p^{r}\right)$ is supersingular if any of the following equivalent properties hold.

1. All Weil numbers of $X$ have the form $\eta_{i}=\sqrt{q} \cdot \zeta_{i}$ where $\zeta_{i}$ is a root of unity.

2. The Newton polygon of $X$ is a straight line of slope $1 / 2$.

3. The Jacobian of $X$ is geometrically isogenous to $E^{g}$ where $E$ is a supersingular elliptic curve.

4. If $X$ has $L$-polynomial $L_{X}(T)=1+\sum_{i=1}^{2 g} c_{i} T^{i}$ then

$$
\operatorname{ord}_{p}\left(c_{i}\right) \geq \frac{i r}{2}, \text { for all } i=1, \ldots, 2 g \text {. }
$$

By the first property, a supersingular curve defined over $\mathbb{F}_{q}$ becomes minimal over some finite extension of $\mathbb{F}_{q}$. Conversely, any minimal or maximal curve is supersingular.

\subsection{Quadratic forms}

We now recall the basic theory of quadratic forms over $\mathbb{F}_{q}$, where $q$ is odd. 
Let $K=\mathbb{F}_{q^{n}}$, and let $Q: K \longrightarrow \mathbb{F}_{q}$ be a quadratic form. The polarization of $Q$ is the symplectic bilinear form $B$ defined by $B(x, y)=Q(x+y)-Q(x)-Q(y)$. By definition the radical of $B$ (denoted $W$ ) is $W=\{x \in K: B(x, y)=0$ for all $y \in K\}$. The rank of $B$ is defined to be $n-\operatorname{dim}(W)$. The rank of $Q$ is defined to be the rank of $B$.

The following result is well known, see Chapter 6 of [5] for example.

Proposition 3. Continue the above notation. Let $N=|\{x \in K: Q(x)=0\}|$, and let $w=\operatorname{dim}(W)$. If $Q$ has odd rank then $N=q^{n-1}$; if $Q$ has even rank then $N=q^{n-1} \pm(q-$ 1) $q^{(n-2+w) / 2}$.

In this paper we will be concerned with quadratic forms of the type $Q(x)=\operatorname{Tr}(f(x))$ where $f(x)$ has the form $\sum a_{i j} x^{p^{i}+p^{j}}$. If $N$ is the number of $x \in \mathbb{F}_{p^{n}}$ with $\operatorname{Tr}(f(x))=0$, then because elements of trace 0 have the form $y^{p}-y$, finding $N$ is equivalent to finding the exact number of

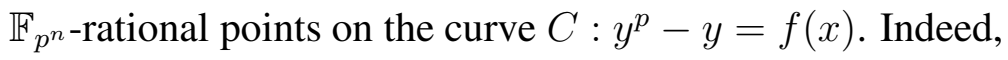

$$
\# C\left(\mathbb{F}_{p^{n}}\right)=p N+1
$$

\subsection{Discrete Fourier Transform}

In this section we recall the statement of the Discrete Fourier Transform and its inverse.

Proposition 4 (Inverse Discrete Fourier Transform). Let $N$ be a positive integer and let $w_{N}$ be a primitive $N$-th root of unity over any field where $N$ is invertible. If

$$
F_{n}=\sum_{j=0}^{N-1} f_{j} w_{N}^{-j n}
$$

for $n=0,1 \cdots, N-1$ then we have

$$
f_{n}=\frac{1}{N} \sum_{j=0}^{N-1} F_{j} w_{N}^{j n}
$$

for $n=0,1 \cdots, N-1$.

\subsection{Relations on the Number of Rational Points}

In this section we state a theorem which allows us to find the number of $\mathbb{F}_{p^{n}}$-rational points of a supersingular curve by finding the the number of $\mathbb{F}_{p^{m}}$-rational points only for the divisors $m$ 
of $s$, where the Weil numbers are $\sqrt{p}$ times an $s$-th root of unity. Note that $s$ is even because equality holds in the Hasse-Weil bound over $\mathbb{F}_{p^{s}}$.

Theorem 1 ([[]]). Let $p$ be an odd prime. Let $X$ be a supersingular curve of genus $g$ defined over $\mathbb{F}_{p}$ whose Weil numbers are $\sqrt{p}$ times an s-th root of unity. Let $n$ be a positive integer, let $\operatorname{gcd}(n, s)=m$ and write $n=m t$. Then we have

$$
\begin{aligned}
&-p^{-n / 2}\left[\# X\left(\mathbb{F}_{p^{n}}\right)-\left(p^{n}+1\right)\right]= \\
& \begin{cases}-p^{-m / 2}\left[\# X\left(\mathbb{F}_{p^{m}}\right)-\left(p^{m}+1\right)\right] & \text { if } m \text { is even, } \\
-p^{-m / 2}\left[\# X\left(F_{q^{m}}\right)-\left(q^{m}+1\right)\right] & \text { if } m \text { is odd and } p \mid t, \\
-p^{-m / 2}\left[\# X\left(\mathbb{F}_{p^{m}}\right)-\left(p^{m}+1\right)\right]\left(\frac{(-1)^{(t-1) / 2} t}{p}\right) & \text { if } m \text { is odd and } p \nmid t,\end{cases}
\end{aligned}
$$

where $(\dot{\bar{p}})$ is the Legendre symbol.

\subsection{A Divisibilty Theorem}

The following theorem is well-known.

Theorem 2. (Kleiman-Serre) If there is a surjective morphism of curves $C \longrightarrow D$ that is defined over $\mathbb{F}_{q}$ then $\mathrm{L}_{D}(T)$ divides $\mathrm{L}_{C}(T)$.

This theorem is sometimes used to show divisibility. The $p=2$ case of Conjecture 1 was proved in [4] by finding a map $C_{k m}^{(2)} \longrightarrow C_{k}^{(2)}$. However, there are cases where there is no map of curves and yet there is divisibility of L-polynomials. We suspect that $C_{k}^{(p)}$ and $C_{2 k}^{(p)}$ is such a case, see Theorem 6, We are unable to find a map $C_{2 k}^{(p)} \longrightarrow C_{k}^{(p)}$ when $p>2$.

\section{The Curve $B_{0}: y^{p}-y=x^{2}$ over $\mathbb{F}_{p}$}

From now on in this paper we will assume that $p$ is an odd prime.

Given a bilinear form $B$ we define

$$
W^{(n)}:=\left\{x \in \mathbb{F}_{p^{n}} \mid B(x, y)=0 \text { for all } y \in \mathbb{F}_{p^{n}}\right\}
$$

In this section we will give the exact number of $\mathbb{F}_{p^{n}}$-rational points on $B_{0}: y^{p}-y=x^{2}$ for all positive integers $n$. Note that $B_{0}$ has genus $(p-1) / 2$. 
Lemma 3. The number of $\mathbb{F}_{p}$-rational points of $B_{0}$ is $p+1$.

Proof. Since $x^{2}=0$ if and only if $x=0$ and since $y^{p}-y=0$ for all $y \in \mathbb{F}_{p}$, we have that the number of $\mathbb{F}_{p}$-rational points of $B_{0}$ (including $\infty$ ) is $p+1$.

Lemma 4. Let $n$ be a positive integer. The radical of the quadratic form $Q_{0}(x)=\operatorname{Tr}\left(x^{2}\right)$ is $\{0\}$ where $\operatorname{Tr}: \mathbb{F}_{p^{n}} \rightarrow \mathbb{F}_{p}$ is the trace map.

Proof. We have

$$
B_{0}(x, y):=Q_{0}(x+y)-Q_{0}(x)-Q_{0}(y)=\operatorname{Tr}(2 x y)
$$

and $W^{(n)}=\{0\}$ because $\operatorname{Tr}(x y)$ is a non-degenerate bilinear form.

Lemma 5. The number of $\mathbb{F}_{p^{2}}$-rational points of $B_{0}$ is

$$
\left\{\begin{array}{lll}
p^{2}+1-(p-1) p & \text { if } p \equiv 1 & \bmod 4 \\
p^{2}+1+(p-1) p & \text { if } p \equiv 3 & \bmod 4
\end{array}\right.
$$

Proof. Since $2-\operatorname{dim}\left(W^{(2)}\right)=2-0=2$ is even by Lemma4, the $N$ in Proposition 3 is equal to $p \pm(p-1)$. By (4) we get

$$
\# B_{0}\left(\mathbb{F}_{p^{2}}\right)=p N+1=p^{2}+1 \pm p(p-1) .
$$

Because the genus of $B_{0}$ is $(p-1) / 2$ we have $2 g \sqrt{p^{2}}=p(p-1)$ and so the curve $B_{0}$ is maximal or minimal over $\mathbb{F}_{p^{2}}$ because the Hasse-Weil bound is met.

Let $\operatorname{Tr}: \mathbb{F}_{p^{2}} \rightarrow \mathbb{F}_{p}$ be the trace map. Then

$$
\operatorname{Tr}\left(x^{2}\right)=x^{2}+x^{2 p}=x^{2}\left(x^{2 p-2}+1\right) .
$$

We know that $B_{0}$ is maximal or minimal over $\mathbb{F}_{p^{2}}$. If it is minimal (resp. maximal), then

$$
\left|\left\{x \in \mathbb{F}_{q^{2}} \mid \operatorname{Tr}\left(x^{2}\right)=0\right\}\right|=1(\text { resp. } 2 p-1) .
$$

In other words, the degree of the greatest common divisor $\left(x^{2 p}+x^{2}, x^{p^{2}}-x\right)$ is

$$
\begin{cases}1 & \text { if } B_{0} \text { is minimal over } \mathbb{F}_{q^{2}}, \\ 2 p-1 & \text { if } B_{0} \text { is maximal over } \mathbb{F}_{q^{2}}\end{cases}
$$


or the degree of the greatest common divisor $\left(x^{2 p-2}+1, x^{p^{2}-1}-1\right)$ is

$$
\begin{cases}0 & \text { if } B_{0} \text { is minimal over } \mathbb{F}_{q^{2}} \\ 2 p-2 & \text { if } B_{0} \text { is maximal over } \mathbb{F}_{q^{2}}\end{cases}
$$

Assume $p \equiv 1 \bmod 4$. Then $(p+1) / 2$ is a positive odd integer and

$$
x^{p^{2}-1}-1=\left(x^{2 p-2}\right)^{(p+1) / 2}-1 \equiv(-1)^{(p+1) / 2}-1=-2 \quad \bmod \left(x^{2 p-2}+1\right)
$$

which implies that $x^{2 p-2}+1$ does not divide $x^{p^{2}-1}-1$. Therefore,

$$
\left(x^{2 p-2}+1, x^{p^{2}-1}-1\right)=1 .
$$

Assume $p \equiv 3 \bmod 4$. Then we have that $(p+1) / 4$ is a positive integer and

$$
x^{p^{2}-1}-1=\left(x^{4 p-4}\right)^{(p+1) / 4}-1
$$

is divisible by $x^{4 p-4}-1$ which equals to $\left(x^{2 p-2}+1\right)\left(x^{2 p-2}-1\right)$. Hence $x^{p^{2}-1}-1$ is divisible by $x^{2 p-2}+1$. Therefore,

$$
\left(x^{2 p-2}+1, x^{p^{2}-1}-1\right)=x^{2 p-2}+1 .
$$

Theorem 6. Let $p \equiv 1 \bmod 4$ and $n \geq 1$ be an integer. Then

$$
-p^{-n / 2}\left[\# B_{0}\left(\mathbb{F}_{p^{n}}\right)-\left(p^{n}+1\right)\right]= \begin{cases}0 & \text { if } n \text { is odd }, \\ p-1 & \text { if } n \text { is even } .\end{cases}
$$

Let $p \equiv 3 \bmod 4$ and $n \geq 1$ be an integer. Then

$$
-p^{-n / 2}\left[\# B_{0}\left(\mathbb{F}_{p^{n}}\right)-\left(p^{n}+1\right)\right]= \begin{cases}0 & \text { if }(4, n)=1, \\ -(p-1) & \text { if }(4, n)=2, \\ p-1 & \text { if }(4, n)=4 .\end{cases}
$$

Proof. It follows by Lemma 3, 5] and Theorem 1, 


\section{The Curve $C_{0}: y^{p}-y=x^{2}+x$ over $\mathbb{F}_{p}$}

In this section we will give the exact number of $\mathbb{F}_{p^{n}}$-rational points on $C_{0}: y^{p}-y=x^{2}+x$ for all positive integer $n$.

Let $n \geq 1$ be a positive integer. The map $(x, y) \rightarrow\left(x-2^{-1}, y\right)$ is a one-to-one map over $\mathbb{F}_{p^{n}}^{2}$. Let $\operatorname{Tr}: \mathbb{F}_{p^{n}} \rightarrow \mathbb{F}_{p}$ be the trace map. Since

$$
\operatorname{Tr}\left(\left(x-2^{-1}\right)^{2}+x\right)=\operatorname{Tr}\left(x^{2}+4^{-1}\right)=\operatorname{Tr}\left(x^{2}\right)+n 4^{-1},
$$

we can use the information on the curve $B_{0}$.

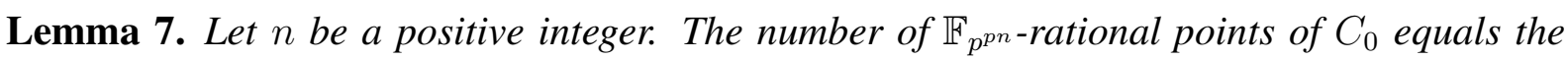

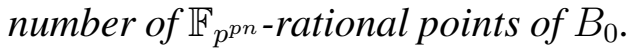

Proof. Let $\operatorname{Tr}: \mathbb{F}_{p^{p n}} \rightarrow \mathbb{F}_{p}$ be the trace map. Since $p \cdot n$ is divisible by $p$, we have

$$
\operatorname{Tr}\left(x^{2}+x\right)=\operatorname{Tr}\left(x^{2}\right) \text {. }
$$

Hence we have the result.

Lemma 8. Let $n$ be a positive integer with $(n, p)=1$. If $\# B_{0}\left(\mathbb{F}_{p^{n}}\right)-\left(p^{n}+1\right) \neq 0$ then

$$
-(p-1)\left(\# C_{0}\left(\mathbb{F}_{p^{n}}\right)-\left(p^{n}+1\right)\right)=\left(\# B_{0}\left(\mathbb{F}_{p^{n}}\right)-\left(p^{n}+1\right)\right) .
$$

Proof. The proof of this lemma is exactly the same as that of Lemma19.

Lemma 9. The number $\# C_{0}\left(\mathbb{F}_{p}\right)$ is $2 p+1$.

Proof. We have $y^{p}-y=0$ for all $y \in \mathbb{F}_{p}$. Also $x^{2}+x=x(x+1)=0$ if and only if $x=0$ or $x=-1$. Therefore, $\# C_{0}\left(\mathbb{F}_{p}\right)=2 \cdot p+1$.

Lemma 10. $C_{0}\left(\mathbb{F}_{p^{2 p}}\right)$ is minimal if $p \equiv 1 \bmod 4$ and maximal $p \equiv 3 \bmod 4$.

Proof. By Lemma 7 we know that $\# C_{0}\left(\mathbb{F}_{p^{2 p}}\right)=\# B_{0}\left(\mathbb{F}_{p^{2 p}}\right)$. Hence it follows by Theorem 6.

We put all these results together in the final Theorem of this section. 
Theorem 11. Let $p \equiv 1 \bmod 4$ and $n \geq 1$ be an integer. Then we have that

$$
-p^{-n / 2}\left[\# C_{0}\left(\mathbb{F}_{p^{n}}\right)-\left(p^{n}+1\right)\right]= \begin{cases}-\left(\frac{n}{p}\right) \sqrt{p} & \text { if }(n, 2 p)=1, \\ -1 & \text { if }(n, 2 p)=2, \\ 0 & \text { if }(n, 2 p)=p, \\ p-1 & \text { if }(n, 2 p)=2 p .\end{cases}
$$

Let $p \equiv 3 \bmod 4$ and $n \geq 1$ be an integer. Then we have that

$$
-p^{-n / 2}\left[\# C_{0}\left(\mathbb{F}_{p^{n}}\right)-\left(p^{n}+1\right)\right]= \begin{cases}-\left(\frac{(-1)^{(n-1) / 2} n}{p}\right) \sqrt{p} & \text { if }(n, 4 p)=1, \\ 1 & \text { if }(n, 4 p)=2, \\ -1 & \text { if }(n, 4 p)=4, \\ 0 & \text { if }(n, 4 p)=p, \\ -(p-1) & \text { if }(n, 4 p)=2 p, \\ p-1 & \text { if }(n, 4 p)=4 p .\end{cases}
$$

Proof. It follows by Lemmas 7, 8, 9, 10 and Theorems 6 and1,

\section{$5 \quad$ The Curve $B_{k}: y^{p}-y=x^{p^{k}+1}$ over $\mathbb{F}_{p}$}

In this section we will give the exact number of $\mathbb{F}_{p^{n}}$-rational points on $B_{k}^{(p)}=B_{k}: y^{p}-y=$ $x^{p^{k}+1}$ for all positive integers $k$ and $n$.

Lemma 12. Let $d \mid k$. The number of $\mathbb{F}_{p^{d}}$-rational points of $B_{k}$ is equal to the number of $\mathbb{F}_{p^{d}}$-rational points of $B_{0}$.

Proof. Since $x^{p^{k}+1}=x^{p^{k}} \cdot x=x \cdot x=x^{2}$ in $\mathbb{F}_{p^{d}}$ for all $d \mid k$, the result is immediate.

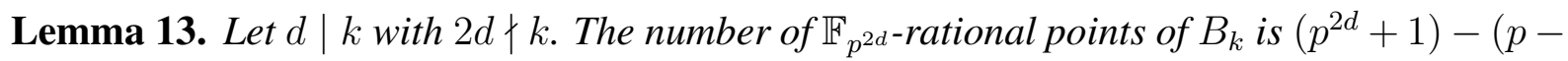
1) $p^{d}$.

Proof. Since $d \mid k$ and $2 d \nmid k$, we have $e:=k / d$ is odd. Define $\operatorname{Tr}_{n}: \mathbb{F}_{p^{n}} \rightarrow \mathbb{F}_{p}$ be the trace 
map. We have

$$
\begin{aligned}
\operatorname{Tr}_{2 d}\left(x^{p^{k}+1}\right) & =\operatorname{Tr}_{d}\left(x^{p^{k}+1}+x^{p^{k+d}+p^{d}}\right) \\
& =\operatorname{Tr}_{d}\left(x^{p^{k}+1}+x^{p^{e d+d}+p^{d}}\right) \\
& =\operatorname{Tr}_{d}\left(x^{p^{e d}+1}+x^{p^{d(e+1)}+p^{d}}\right) \\
& =\operatorname{Tr}_{d}\left(x^{p^{d}+1}+x^{p^{d(e+1)}+p^{d}}\right) \\
& =\operatorname{Tr}_{d}\left(x^{p^{d}+1}+x^{1+p^{d}}\right) \\
& =\operatorname{Tr}_{d}\left(2 x^{p^{d}+1}\right) .
\end{aligned}
$$

Since $x \rightarrow x^{p^{d}+1}$ is $p^{d}+1$-to-1 map from $\mathbb{F}_{p^{2 d}}^{\times}$to $\mathbb{F}_{p^{d}}^{\times}$and since $\operatorname{Tr}_{d}(x)$ is a linear map from $\mathbb{F}_{p^{d}}$ to $\mathbb{F}_{p}$, we have that the number of $\mathbb{F}_{p^{2 d}}$-rational points of $B_{k}$ is

$$
1+p\left(1+\left(p^{d}+1\right)\left(p^{d-1}-1\right)\right)=\left(p^{2 d}+1\right)-(p-1) p^{d} .
$$

Lemma 14. The curve $B_{k}$ is minimal over $\mathbb{F}_{p^{4 k}}$.

Proof. Define $Q_{k}(x)=\operatorname{Tr}\left(x^{p^{k}+1}\right)$ where $\operatorname{Tr}: \mathbb{F}_{p^{n}} \rightarrow \mathbb{F}_{p}$ is the trace map $(n=4 k)$. We have

$$
B(x, y):=Q(x+y)-Q(x)-Q(y)=\operatorname{Tr}\left(x^{p^{k}} y+x y^{p^{k}}\right)=\operatorname{Tr}\left(y^{p^{k}}\left(x^{p^{2 k}}+x\right)\right)
$$

and

$$
W^{(n)}:=\left\{x \in \mathbb{F}_{p^{n}} \mid B(x, y)=0 \text { for all } y \in \mathbb{F}_{p^{n}}\right\}=\left\{x \in \mathbb{F}_{p^{n}} \mid x^{p^{2 k}}+x=0\right\} .
$$

So $W^{(4 k)} \subseteq \mathbb{F}_{p^{4 k}}$ and so the rank of $Q_{k}$ is $n-\operatorname{dim} W^{(n)}=4 k-2 k$ which is even, and so the $N$ in Proposition 3 is equal to $p^{n-1} \pm(p-1) p^{3 k-1}$. By (4) we get

$$
\# B_{0}\left(\mathbb{F}_{p^{4 k}}\right)=p N+1=p^{n}+1 \pm(p-1) p^{3 k} .
$$

The genus of $B_{k}$ is $p^{k}(p-1) / 2$ so $2 g \sqrt{p^{4 k}}=p^{3 k}(p-1)$, and so the curve $B_{k}$ is maximal or minimal over $\mathbb{F}_{p^{4 k}}$ because the Hasse-Weil bound is met.

If the curve $B_{k}$ is maximal over $\mathbb{F}_{p^{4 k}}$, then $\# B_{k}\left(\mathbb{F}_{p^{2 k}}\right)$ has to be $p^{2 k}+1$ by Proposition 2 , However, $W^{(2 k)}=\{0\}$ and so $2 k-\operatorname{dim} W^{(2 k)}=2 k-0=2 k$ is even, which means that $\# B_{k}\left(\mathbb{F}_{p^{2 k}}\right)$ cannot be $p^{2 k}+1$ by Proposition 3 . Hence the curve $B_{k}$ is minimal over $\mathbb{F}_{p^{4 k}}$.

Corollary 1. We have $\zeta^{4 k}=1$ for all $\zeta$ where $\sqrt{q} \zeta$ is a Weil number of $B_{k}$.

Proof. We have shown that $B_{k}$ is minimal over $\mathbb{F}_{p^{4 k}}$, and it follows from Sections 2.1 and 2.2 . 


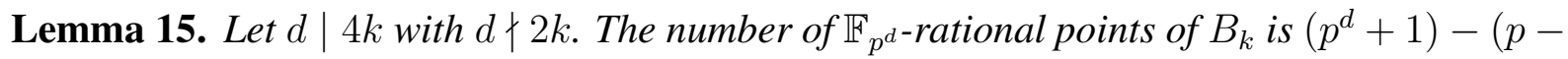
1) $p^{3 d / 4}$.

Proof. Since $d \mid 4 k$ and $d \nmid 2 k$, we have $e:=4 k / d$ is odd and $d=4 f$ for some integer $f$. Define $\operatorname{Tr}_{n}: \mathbb{F}_{p^{n}} \rightarrow F_{p}$ be the trace map. We have

$$
\begin{aligned}
\operatorname{Tr}_{d}\left(x^{p^{k}+1}\right) & =\operatorname{Tr}_{f}\left(x^{p^{k}+1}+x^{p^{k+f}+p^{f}}+x^{p^{k+2 f}+p^{2 f}}+x^{p^{k+3 f}+p^{3 f}}\right) \\
& =\operatorname{Tr}_{f}\left(x^{p^{e f}+1}+x^{p^{f(e+1)}+p^{f}}+x^{p^{f(e+2)}+p^{2 f}}+x^{p^{f(e+3)}+p^{3 f}}\right) \\
& =\operatorname{Tr}_{f}\left(x^{p^{f}+1}+x^{p^{2 f}+p^{f}}+x^{p^{3 f}+p^{2 f}}++x^{p^{4 f}+p^{3 f}}\right) \\
& =\operatorname{Tr}_{d}\left(x^{p^{f}+1}\right) .
\end{aligned}
$$

Since $4 f=d \mid d$, the curve $B_{f}$ is minimal over $\mathbb{F}_{p^{d}}$ and hence The number of $\mathbb{F}_{p^{d}}$-rational points of $B_{k}$ is

$$
\left(p^{d}+1\right)-(p-1) p^{3 d / 4}
$$

Corollary 2. If $d \mid k$ and $d$ is odd, then

$$
\# B_{k}\left(\mathbb{F}_{p^{d}}\right)=p^{d}+1
$$

If $d \mid k$ and $d$ is even, then

$$
\# B_{k}\left(\mathbb{F}_{p^{d}}\right)= \begin{cases}\left(p^{d}+1\right)+(p-1) p^{d / 2} & \text { if } 2 \| d \text { and } p \equiv 3 \bmod 4 \\ \left(p^{d}+1\right)-(p-1) p^{d / 2} & \text { if } 4 \mid d \text { or } p \equiv 1 \quad \bmod 4 .\end{cases}
$$

If $d \nmid k$ and $\frac{d}{2} \mid k$, then

$$
\# B_{k}\left(\mathbb{F}_{p^{d}}\right)=p^{d}+1-(p-1) p^{d / 2}
$$

If $d \nmid 2 k$ and $d \mid 4 k$, then

$$
\# B_{k}\left(\mathbb{F}_{p^{d}}\right)=\left(p^{d}+1\right)-(p-1) p^{3 d / 4}
$$

We put all these results together.

Theorem 16. Let $n \geq 1$ be an integer and let $d=(n, 4 k)$. Then we have that

$$
-p^{-n / 2}\left[\# B_{k}\left(\mathbb{F}_{p^{n}}\right)-\left(p^{n}+1\right)\right]= \begin{cases}0 & \text { if } d \mid k \text { and } d \text { is } \text { odd }, \\ (-1)^{n(p-1) / 4}(p-1) & \text { if } d \mid k \text { and } d \text { is even, } \\ -(p-1) & \text { if } d \nmid k \text { and } \frac{d}{2} \mid k, \\ (p-1) p^{(k, n)} & \text { if } d \nmid 2 k \text { and } d \mid 4 k .\end{cases}
$$

Proof. It follows by Corollary 2 and Theorem 1 . 


\section{The Curve $C_{k}: y^{p}-y=x^{p^{k}+1}+x$ over $\mathbb{F}_{p}$}

In this section we will give the exact number of $\mathbb{F}_{p^{n}}$-rational points on $C_{k}: y^{p}-y=x^{p^{k}+1}+x$ for all positive integers $k$ and $n$.

Let $(x, y) \rightarrow\left(x-2^{-1}, y\right)$ is a one-to-one map over $\mathbb{F}_{p^{n}}^{2}$. Since

$$
\begin{aligned}
\operatorname{Tr}\left(\left(x-2^{-1}\right)^{p^{k}+1}+\left(x-2^{-1}\right)\right) & =\operatorname{Tr}\left(x^{p^{k}+1}-2^{-1} x^{p^{k}}+2^{-1} x-4^{-1}\right) \\
& =\operatorname{Tr}\left(x^{p^{k}+1}\right)-n 4^{-1},
\end{aligned}
$$

we can use the information on the curve $B_{k}$.

Lemma 17. If $p \mid n$, the number of $\mathbb{F}_{p^{n}}$-rational points of $C_{k}$ equals the number of $\mathbb{F}_{p^{n}}$-rational points of $B_{k}$.

Proof. Since $n$ is divisible by $p$, by (6) we have

$$
\left|\left\{x \in \mathbb{F}_{p^{n}}: \operatorname{Tr}\left(x^{p^{k}+1}+x\right)=0\right\}\right|=\mid\left\{x \in \mathbb{F}_{p^{n}}: \operatorname{Tr}\left(x^{p^{k}+1}\right)=0\right\} .
$$

Hence we have the result.

Corollary 3. If $p \mid k$ we have $\zeta^{4 k}=1$ for all $\zeta$ where $\sqrt{q} \zeta$ is a Weil number of $C_{k}$.

Proof. Follows from Lemma 17 and Corollary 1.

Remark. It follows from Lemma 17 that $C_{k}$ and $B_{k}$ have the same L-polynomial when considered as curves defined over $\mathbb{F}_{p^{p}}$. They do not have the same L-polynomial when considered as curves defined over $\mathbb{F}_{p}$, as the results in this paper show (see Lemma 19 below). This means that the $p$-th powers of the Weil numbers of $B_{k}$ and $C_{k}$ (considered as curves defined over $\mathbb{F}_{p}$ ) are equal, but the Weil numbers themselves are not the same. For example, the L-polynomial of $B_{2}^{(3)}$ is

$$
19683 T^{18}+6561 T^{16}-486 T^{10}-162 T^{8}+3 T^{2}+1
$$

and the L-polynomial of $C_{2}^{(3)}$ is

$$
19683 T^{18}-19683 T^{17}+6561 T^{16}+243 T^{10}-243 T^{9}+81 T^{8}+3 T^{2}-3 T+1 .
$$

For both of these, the polynomial whose roots are the cubes of the roots is

$$
1+27 T^{2}-1062882 T^{8}-28697814 T^{10}+282429536481 T^{16}+7625597484987 T^{18}
$$

which is the L-polynomial of both $B_{2}$ and $C_{2}$ considered as curves defined over $\mathbb{F}_{3^{3}}$. 
Lemma 18. Let $d \mid k$. The number of $\mathbb{F}_{p^{d}}$-rational points of $C_{k}$ is the number of $\mathbb{F}_{p^{d}}$-rational points of $C_{0}$.

Proof. Since

$$
x^{p^{k}+1}+x=x^{p^{k}} \cdot x+x=x \cdot x+x=x^{2}+x
$$

in $\mathbb{F}_{p^{d}}$ for all $d \mid k$, the result is immediate.

Lemma 19. Let $n$ be a positive integer with $(n, p)=1$. If $\# B_{k}\left(\mathbb{F}_{p^{n}}\right)-\left(p^{n}+1\right) \neq 0$ then

$$
-(p-1)\left(\# C_{k}\left(\mathbb{F}_{p^{n}}\right)-\left(p^{n}+1\right)\right)=\left(\# B_{k}\left(\mathbb{F}_{p^{n}}\right)-\left(p^{n}+1\right)\right) .
$$

Proof. Let $b:=n 4^{-1} \neq 0$, let

$$
N_{0}=\left|\left\{x \in \mathbb{F}_{p^{n}}: \operatorname{Tr}\left(x^{p^{k}+1}\right)=0\right\}\right|
$$

and let

$$
N_{1}=\left|\left\{x \in \mathbb{F}_{p^{n}}: \operatorname{Tr}\left(x^{p^{k}+1}\right)=b\right\}\right| .
$$

By (6) and also (4) we get $\# C_{k}\left(\mathbb{F}_{p^{n}}\right)=p N_{1}+1$. So

$$
\# C_{k}\left(\mathbb{F}_{p^{n}}\right)-\left(p^{n}+1\right)=p N_{1}-p^{n}
$$

The nonzero values of the quadratic form $\operatorname{Tr}\left(x^{p^{k}+1}\right)$ are evenly distributed over the nonzero elements of $\mathbb{F}_{p}$ by [5, Theorem 6.26], so

$$
N_{0}+(p-1) N_{1}=p^{n}
$$

Substituting for $N_{1}$ into (7) gives

$$
\# C_{k}\left(\mathbb{F}_{p^{n}}\right)-\left(p^{n}+1\right)=\frac{p^{n+1}-p N_{0}}{p-1}-p^{n}
$$

or

$$
(p-1)\left(\# C_{k}\left(\mathbb{F}_{p^{n}}\right)-\left(p^{n}+1\right)\right)=p^{n}-p N_{0}
$$

Finally, by (4) again we note that

$$
\# B_{k}\left(\mathbb{F}_{p^{n}}\right)-\left(p^{n}+1\right)=p N_{0}-p^{n} .
$$


Corollary 4. If $p \nmid k$ we have $\zeta^{4 k p}=1$ for all $\zeta$ where $\sqrt{q} \zeta$ is a Weil number of $C_{k}$.

Proof. The previous lemma shows that that $C_{k}$ is minimal over $\mathbb{F}_{p^{4 k p}}$, and it follows from Sections 2.1 and 2.2.

Corollary 5. Let $k$ be a positive integer. Define

$$
l= \begin{cases}k & \text { if } p \mid k, \\ k p & \text { if } p \nmid k .\end{cases}
$$

If $d \mid l$ and $d$ is odd and relatively prime to $p$, then

$$
\# C\left(\mathbb{F}_{p^{d}}\right)=p^{d}+1+\left(\frac{(-1)^{(d-1) / 2} d}{p}\right) p^{(d+1) / 2} .
$$

If $d \mid l$ and $d$ is odd and divisible by $p$, then

$$
\# C_{k}\left(\mathbb{F}_{p^{d}}\right)=0
$$

If $d \mid l$ and $d$ is even and relatively prime to $p$, then

$$
\# C_{k}\left(\mathbb{F}_{p^{d}}\right)= \begin{cases}\left(p^{d}+1\right)-p^{d / 2} & \text { if } 2 \| d \text { and } p \equiv 3 \bmod 4 \\ \left(p^{d}+1\right)+p^{d / 2} & \text { if } 4 \mid d \text { or } p \equiv 1 \bmod 4 .\end{cases}
$$

If $d \mid l$ and $d$ is even and divisible by $p$, then

$$
\# C_{k}\left(\mathbb{F}_{p^{d}}\right)= \begin{cases}\left(p^{d}+1\right)+(p-1) p^{d / 2} & \text { if } 2 \| d \text { and } p \equiv 3 \bmod 4 \\ \left(p^{d}+1\right)-(p-1) p^{d / 2} & \text { if } 4 \mid d \text { or } p \equiv 1 \bmod 4\end{cases}
$$

If $d \nmid l$ and $\frac{d}{2} \mid l$ and $d$ is relatively prime to $p$, then

$$
C_{k}\left(\mathbb{F}_{p^{d}}\right)=\left(p^{d}+1\right)+p^{d / 2} .
$$

If $d \nmid l$ and $\frac{d}{2} \mid l$ and $d$ is relatively prime to $p$, then

$$
C_{k}\left(\mathbb{F}_{p^{d}}\right)=\left(p^{d}+1\right)-(p-1) p^{d / 2} .
$$

If $d \nmid 2 l, d \mid 4 l$ and $d$ is relatively prime to $p$, then

$$
\# C_{k}\left(\mathbb{F}_{p^{d}}\right)=\left(p^{d}+1\right)+p^{3 d / 4} .
$$

If $d \nmid 2 l, d \mid 4 l$ and $d$ is divisible by $p$, then

$$
\# C_{k}\left(\mathbb{F}_{p^{d}}\right)=\left(p^{d}+1\right)-(p-1) p^{3 d / 4} .
$$


Theorem 20. Let $k$ be a positive integer. Define

$$
l= \begin{cases}k & \text { if } p \mid k, \\ k p & \text { if } p \nmid k .\end{cases}
$$

Let $n \geq 1$ be an integer with $d=(n, 4 l)$. Then we have

$$
-p^{-n / 2}\left[\# C_{k}\left(\mathbb{F}_{p^{n}}\right)-\left(p^{n}+1\right)\right]= \begin{cases}-\left(\frac{(-1)^{(n-1) / 2} n}{p}\right) \sqrt{p} & \text { if } d \mid l \text { and } n \text { is odd and } p \nmid n, \\ 0 & \text { if } d \mid l \text { and } n \text { is odd and } p \mid n, \\ -(-1)^{n(p-1) / 4} & \text { if } d \mid l \text { and } n \text { is } \text { even and } p \nmid n, \\ (-1)^{n(p-1) / 4}(p-1) & \text { if } d \mid l \text { and } n \text { is } \text { even and } p \mid n, \\ -1 & \text { if } d \nmid l \text { and } \frac{d}{2} \mid l \text { and } p \nmid n, \\ p-1 & \text { if } d \nmid l \text { and } \frac{d}{2} \mid l \text { and } p \mid n, \\ -p^{(k, n)} & \text { if } d \nmid 2 l \text { and } d \mid 4 l \text { and } p \nmid n, \\ (p-1) p^{(k, n)} & \text { if } d \nmid 2 l \text { and } d \mid 4 l \text { and } p \mid n .\end{cases}
$$

Proof. It follows by Corollary 5 and Theorem 1 .

\section{Divisibility Property of the Curves $C_{k}$}

In this section we will prove Conjecture1, The proof will be broken into a few parts. The first part is to show that the L-polynomial of $C_{k}$ divides the L-polynomial of $C_{2 k}$. The next part is to show that the L-polynomial of $C_{k}$ divides the L-polynomial of $C_{t k}$ where $t$ is odd. Finally, these results are combined to prove the conjecture.

Lemma 21. Let $k$ be a positive integer. Define

$$
s= \begin{cases}8 k & \text { if } p \mid k, \\ 8 k p & \text { if } p \nmid k .\end{cases}
$$

For $n \geq 1$ define

$$
U_{n}=-p^{-n / 2}\left[\# C_{2 k}\left(\mathbb{F}_{p^{n}}\right)-\# C_{k}\left(\mathbb{F}_{p^{n}}\right)\right]
$$

and write $U_{n}$ as a linear combination of the s-th roots of unity as

$$
U_{n}=\sum_{j=0}^{s-1} u_{j} w_{s}^{-j n} .
$$


Then we have

$$
u_{n} \geq 0
$$

for all $n \in\{0,1, \cdots, s-1\}$.

Proof. Let $U_{0}=U_{s}$. Write $k=2^{v} t$ where $v$ is a positive integer and $t$ is an odd integer. By Theorem 20 we have

$$
U_{n}= \begin{cases}0 & \text { if } 2^{v+1} \nmid n, \\ -1+(-1)^{n(p-1) / 4} & \text { if } 2^{v+1} \| n \text { and } p \nmid n, \\ \left.(p-1)\left(1-(-1)^{n(p-1) / 4}\right)\right) & \text { if } 2^{v+1}|| n \text { and } p \mid n, \\ p^{(k, n)}-1 & \text { if } 2^{v+2} \| n \text { and } p \nmid n, \\ -(p-1)\left(p^{(k, n)}-1\right) & \text { if } 2^{v+2} \| n \text { and } p \mid n, \\ -\left(p^{(2 k, n)}-p^{(k, n)}\right) & \text { if } 2^{v+3} \mid n \text { and } p \nmid n, \\ (p-1)\left(p^{(2 k, n)}-p^{(k, n)}\right) & \text { if } 2^{v+3} \mid n \text { and } p \mid n .\end{cases}
$$

If $k$ is divisible by $p$, by using Inverse Discrete Fourier Transform we have

$$
\begin{aligned}
u_{n} & =\frac{1}{8 k} \sum_{j=0}^{8 k-1} U_{j} w_{8 k}^{j n} \\
& =\frac{1}{8 k} \sum_{j=0}^{4 t-1} U_{2^{v+1} j} w_{2 t}^{j n} \quad \text { because } U_{n}=0 \text { if } 2^{v+1} \nmid n \\
& \geq \frac{1}{8 k}\left(U_{0}-\sum_{j=1}^{4 t-1}\left|U_{2^{v+2} j}\right|\right) \quad \text { by the triangle inequality } \quad \text { and all others are } \leq p^{k}(p-1) \\
& \geq \frac{1}{8 k}\left[(p-1)\left(p^{2 k}-p^{k}\right)-(4 t-1)(p-1) p^{k}\right] \quad \text { because } U_{s}=U_{0}=(p-1)\left(p^{2 k}-p^{k}\right) \\
& =\frac{1}{8 k}(p-1) p^{k}\left(p^{k}-4 t\right) \\
& \geq \frac{1}{8 k}(p-1) p^{k}\left(p^{k}-4 k\right) \\
& \geq 0 .
\end{aligned}
$$


If $k$ and $n$ are not divisible by $p$, by using the Inverse Discrete Fourier Transform we have

$$
\begin{aligned}
u_{n} & =\frac{1}{8 k p} \sum_{j=0}^{8 k p-1} U_{j} w_{8 k p}^{j n} \\
& =\frac{1}{8 k p} \sum_{j=0}^{4 t p-1} U_{2^{v+1} j} w_{2 t p}^{j n} \\
& \geq \frac{1}{8 k p}\left(U_{0}+\sum_{j=1}^{p-1} U_{2^{v+3} t j} w_{p}^{j}-\sum_{j=0,2 t \nmid j}^{2 t p-1}\left|U_{2^{v+2} j}\right|-\sum_{j=0}^{2 t p-1}\left|U_{2^{v+1}(2 j+1)}\right|\right) .
\end{aligned}
$$

We have

$$
\left|U_{2^{v+1}(2 j+1)}\right| \leq \begin{cases}2 & \text { if } p \nmid(2 j+1), \\ 2(p-1) & \text { if } p \mid(2 j+1) .\end{cases}
$$

Since there are $2 t$ (resp. $2 t(p-1)$ ) integers which is (resp. not) divisible by $p$ between 0 and $2 t p-1$, we have

$$
\sum_{j=0}^{2 t p-1}\left|U_{2^{v+1}(2 j+1)}\right| \leq 2 t \cdot 2(p-1)+2 t(p-1) \cdot 2=8 t(p-1) .
$$

Therefore, we have

$$
\begin{aligned}
u_{n} & \geq \frac{1}{8 k p}\left[(p-1)\left(p^{2 k}-p^{k}\right)+\left(p^{2 k}-p^{k}\right)-(2 t p-p)(p-1) p^{k}-8 t(p-1)\right] \\
& \geq \frac{1}{8 k} p^{k}\left(p^{k}-(p-1)(2 t-1)\right)-\frac{p-1}{p} \\
& >-1 .
\end{aligned}
$$

Since $u_{n}$ is an integer, we have $u_{n} \geq 0$.

Assume $k$ is not divisible by $p$ and $n$ is divisible by $p$ and write $n=m p$. We will show that $u_{n}=0$. By the Inverse Discrete Fourier Transform we have

$$
\begin{aligned}
u_{n} & =\frac{1}{8 k p} \sum_{j=0}^{8 k p-1} U_{j} w_{8 k p}^{j n} \\
& =\frac{1}{8 k p} \sum_{j=0}^{4 t p-1} U_{2^{v+1} j} w_{4 t}^{j m} \\
& =\frac{1}{8 k p} \sum_{j=0}^{4 t-1}\left[\left(\sum_{i=0}^{p-1} U_{2^{v+1}(4 t i+j)}\right) w_{2 t}^{j m}\right] .
\end{aligned}
$$


Since $(4 t, p)=1$, for any integer $j$ we have

$$
\{4 t i+j \bmod p \mid 0 \leq i \leq p-1\}=\{i \bmod p \mid 0 \leq i \leq p-1\}
$$

and so exactly one of the $4 t i+j$ is divisible by $p$. Therefore, for each $j$,

$$
\sum_{i=0}^{p-1} U_{2^{v+2}(2 t i+j)}=0
$$

because if $j$ is odd then one term is $(p-1)\left(1-(-1)^{n(p-1) / 4}\right)$ and the other $p-1$ terms are $-1+(-1)^{n(p-1) / 4}$, if $2 \| j$ then one term is $-(p-1)\left(p^{(k, n)}-1\right)$ and the other $p-1$ terms are $p^{(k, n)}-1$, and if $4 \mid j$ is even then one term is $(p-1)\left(p^{(2 k, n)}-p^{(k, n)}\right)$ and the other $p-1$ terms are $-\left(p^{(2 k, n)}-p^{(k, n)}\right)$.

We write $L\left(C_{k}\right)$ for $L_{C_{k}}$.

Corollary 6. Let $k$ be a positive integer. Then

$$
L\left(C_{k}\right) \mid L\left(C_{2 k}\right)
$$

Proof. Lemma 21 shows that the multiplicity of each root of $L\left(C_{k}\right)$ is smaller than or equal to its multiplicity as a root of $L\left(C_{2 k}\right)$.

Lemma 22. Let $k$ be an integer and $t$ be an odd integer. Then

$$
L\left(C_{k}\right) \mid L\left(C_{k t}\right)
$$

Proof. Let $X_{k}: y^{p}-y=x^{p^{k}+1}-4^{-1}$ over $\mathbb{F}_{p}$ and check that

$$
(x, y) \mapsto\left(x-\frac{1}{2}, y-\frac{1}{2} \sum_{i=0}^{k-1} x^{p^{i}}\right)
$$

is a map $X_{k} \longrightarrow C_{k}$. The map is defined everywhere and is invertible, so $C_{k}$ is isomorphic to $X_{k}$, and hence $L\left(C_{k}\right)=L\left(X_{k}\right)$. Therefore, it is enough to show that $L\left(X_{k}\right) \mid L\left(X_{k t}\right)$.

Since $t$ is odd, $p^{k}+1$ divides $p^{k t}+1$ and therefore there is a map of curves $X_{k t} \longrightarrow X_{k}$ given by $(x, y) \rightarrow\left(x^{\left(p^{k t}+1\right) /\left(p^{k}+1\right)}, y\right)$. Hence $L\left(X_{k}\right) \mid L\left(X_{k t}\right)$ by Theorem 2 .

Theorem 23. Let $k$ and $m$ be positive integers. Then

$$
L\left(C_{k}\right) \mid L\left(C_{k m}\right)
$$


Proof. If $m=1$, then the result is trivial. Assume $m \geq 2$ and write $m=2^{s} t$ where $t$ is odd. Since $t$ is odd, by Lemma 22 we have

$$
L\left(C_{k}\right) \mid L\left(C_{k t}\right)
$$

and by Corollary 6 we have

$$
L\left(C_{2^{i-1} k t}\right) \mid L\left(C_{2^{i} k t}\right)
$$

for all $i \in\{1, \cdots, s\}$. Hence

$$
L\left(C_{k}\right) \mid L\left(C_{k m}\right) .
$$

\section{Remark on the Divisibility Property of the Curves $B_{k}$}

The divisibility property of the curves $B_{k}$ can be proved in the same way as for $C_{k}$. For odd $t$, we have a natural map from $B_{t k}$ to $B_{k}$ which sends $(x, y)$ to $\left(x^{\left(p^{k t}+1\right) /\left(p^{k}+1\right)}, y\right)$. We are unable to find a map from $B_{2 k}$ to $B_{k}$, so we use a similar argument. For $n \geq 1$ we can define

$$
U_{n}=-p^{-n / 2}\left[\# B_{2 k}\left(\mathbb{F}_{p^{n}}\right)-\# B_{k}\left(\mathbb{F}_{p^{n}}\right)\right]= \begin{cases}0 & \text { if } 2^{v+1} \nmid n, \\ \left.(p-1)\left(1-(-1)^{n(p-1) / 4}\right)\right) & \text { if } 2^{v+1} \| n \text { and } p \mid n, \\ -(p-1)\left(p^{(k, n)}-1\right) & \text { if } 2^{v+2} \| n \text { and } p \mid n, \\ (p-1)\left(p^{(2 k, n)}-p^{(k, n)}\right) & \text { if } 2^{v+3} \mid n \text { and } p \mid n .\end{cases}
$$

and can similarly show that $u_{j} \geq 0$ for all $j \in\{0, \cdots, 4 k-1\}$ where

$$
U_{n}=\sum_{j=0}^{4 k-1} u_{j} w_{4 k}^{-j n}
$$

We write $L\left(B_{k}\right)$ for $L_{B_{k}}$.

Theorem 24. Let $k$ and $m$ be positive integers. Then

$$
L\left(B_{k}\right) \mid L\left(B_{k m}\right)
$$

The proof is similar to the proof of Theorem 23 , 


\section{Opposite Direction}

In this section, we will prove that the opposite directions of the divisibility theorems for $B_{k}$ and $C_{k}$ are also valid. To be precise, we have shown that if $k$ divides $\ell$ then the L-polynomial of $C_{k}$ (or $B_{k}$ ) divides the L-polynomial of $C_{\ell}$ (or $B_{\ell}$ ). We now prove that if $k$ does not divide $\ell$ then the L-polynomials do not divide.

Let $X$ be a supersingular curve defined over $\mathbb{F}_{q}$. The smallest positive integer $s=s_{X}$ such that $\zeta_{i}^{s}=1$ for all $i=1, \ldots, 2 g$ will be called the period of $X$. The period depends on $q$, in the sense that $X\left(\mathbb{F}_{q^{n}}\right)$ may have a different period to $X\left(\mathbb{F}_{q}\right)$.

Proposition 5. Let $C$ and $D$ be supersingular curves over $\mathbb{F}_{q}$. If $L(C)$ divides $L(D)$, then $s_{C}$ divides $s_{D}$.

Proof. Since $L(C)$ divides $L(D)$, the roots of $L(C)$ are also roots of $L(D)$. Therefore, any Weil number of $C$ is also a Weil number of $D$. Let $\sqrt{q} \zeta$ be a Weil number for $C$. Since $\zeta^{s_{D}}=1$, the order of $\zeta$ divides $s_{D}$. Since this happens for all Weil numbers of $C, s_{C}$ divides $s_{D}$.

Theorem 25. Let $k$ and $\ell$ be positive integers such that $k$ does not divide $\ell$. Then $L\left(B_{k}\right)$ does not divide $L\left(B_{\ell}\right)$.

Proof. By Corollary 1 the period of $B_{k}$ is $4 k$ and the period of $B_{\ell}$ is $4 \ell$. Since $4 k$ does not divide $4 \ell$, we have $L\left(B_{k}\right)$ does not divide $L\left(B_{\ell}\right)$ by Proposition 5 ,

Corollary 7. Let $k$ and $\ell$ be positive integers such that $k<\ell$ and $k$ does not divide $\ell$. Then there is no map from $B_{\ell}$ to $B_{k}$.

Proof. By the Kleiman-Serre theorem (Theorem 2) and Theorem 25 .

Now we turn to $C_{k}$.

Lemma 26. Let $\ell$ be a positive integer coprime to $p$. Then $L\left(C_{p}\right)$ does not divide $L\left(C_{\ell}\right)$.

Proof. We will check the multiplicities of $T-\sqrt{p}$ in $L_{C_{p}}(T)$ and $L_{C_{\ell}}(T)$.

Using the Inverse Fourier Transform, the multiplicity of $T-\sqrt{p}$ in $L_{C_{p}}(T)$ is

$$
\frac{1}{4 p} \sum_{j=1}^{4 p}\left(p^{-j / 2}\left[\# C_{p}\left(\mathbb{F}_{p^{j}}\right)-\left(p^{j}+1\right)\right]\right) \geq \frac{1}{4 p}\left[(p-1) p^{p}-(4 p-1) p\right]>0
$$


by Theorem 20 and triangle inequality.

Using the Inverse Fourier Transform, the multiplicity of $T-\sqrt{p}$ in $L_{C_{\ell}}(T)$ is

$$
\frac{1}{4 p \ell} \sum_{j=1}^{4 p \ell}\left(p^{-j / 2}\left[\# C_{\ell}\left(\mathbb{F}_{p^{j}}\right)-\left(p^{j}+1\right)\right]\right)
$$

which is 0 because

$\mid\{k \in \mathbb{Z} \mid(4 p \ell, k)=d$ and $1 \leq j \leq 4 p \ell\}|=(p-1) \cdot|\{k \in \mathbb{Z} \mid(4 p \ell, k)=d p$ and $1 \leq j \leq 4 p \ell\} \mid$ for any $d \mid l$ and by Theorem 20 .

Theorem 27. Let $k$ and $\ell$ be positive integers such that $k$ does not divide $\ell$. Then $L\left(C_{k}\right)$ does not divide $L\left(C_{\ell}\right)$.

Proof. We use Corollaries 3 and 4 which give the period of $C_{k}$.

Case I: If $p \mid k, l$, then the period of $C_{k}$ is $4 k$ and the period of $C_{\ell}$ is $4 \ell$. Since $4 k \nmid 4 \ell, L\left(C_{k}\right)$ does not divide $L\left(C_{\ell}\right)$ by Proposition 5 .

Case II: If $p \nmid k$ and $p \mid \ell$, then the period of $C_{k}$ is $4 k p$ and the period of $C_{\ell}$ is $4 \ell$. Since $4 k p \nmid 4 \ell$, $L\left(C_{k}\right)$ does not divide $L\left(C_{\ell}\right)$ by Proposition 5 .

Case III: If $p \nmid k$ and $p \nmid \ell$, then the period of $C_{k}$ is $4 k p$ and the period of $C_{\ell}$ is $4 \ell p$. Since $4 k p \nmid 4 \ell p, L\left(C_{k}\right)$ does not divide $L\left(C_{\ell}\right)$ by Proposition 5 .

Case IV - A: If $p \mid k, p \nmid \ell$ and $(k / p) \nmid \ell$, then the period of $C_{k}$ is $4 k$ and the period of $C_{\ell}$ is $4 \ell p$. Since $4 k \nmid 4 \ell p, L\left(C_{k}\right)$ does not divide $L\left(C_{\ell}\right)$ by Proposition 5 .

Case IV - B: If $p \mid k, p \nmid \ell$ and $(k / p) \mid \ell$, then the period of $C_{k}$ is $4 k$ and the period of $C_{\ell}$ is $4 \ell p$. Since $4 k \mid 4 \ell p$, we cannot use the Proposition 5 .

Since $p \mid k$, we have $L\left(C_{p}\right)$ divides $L\left(C_{k}\right)$. If $L\left(C_{k}\right)$ divides $L\left(C_{\ell}\right)$, then $L\left(C_{p}\right)$ divides $L\left(C_{\ell}\right)$ which gives a contradiction by Lemma 26 .

Corollary 8. Let $k$ and $\ell$ be positive integers such that $k<\ell$ and $k$ does not divide $\ell$. Then there is no map from $C_{\ell}$ to $C_{k}$.

Proof. By the Kleiman-Serre theorem (Theorem 2) and Theorem 27. 
We close this section by remarking again that we do not know if there is a rational map from $B_{2 k}$ to $B_{k}$ or from $C_{2 k}$ to $C_{k}$.

\section{Remark on the the Curves $y^{p}-y=x^{p^{k}+1}+a x$ over $\mathbb{F}_{p}$}

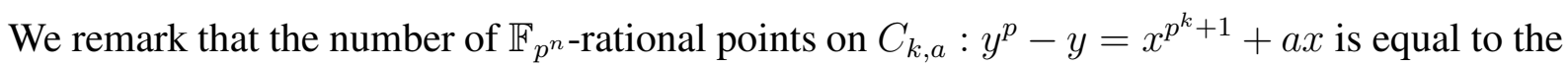
number of $\mathbb{F}_{p^{n}}$-rational points on $C_{k}$, where $a \in \mathbb{F}_{p}^{\times}$.

All the proofs in this paper go through, with minor changes. There is the same relationship to $B_{k}$, the map in (6) must be changed to the map $(x, y) \mapsto\left(x-a 2^{-1}, y\right)$ and all proofs go through. The curve $X_{k}$ in the proof of Lemma 22 must be replaced by $y^{p}-y=x^{p^{k}+1}-a 4^{-1}$.

Therefore, the divisibility property (and its opposite direction) also holds for these curves.

\section{References}

[1] Irene Bouw, Wei Ho, Beth Malmskog, Renate Scheidler, Padmavathi Srinivasan, and Christelle Vincent, Zeta functions of a class of Artin-Schreier curves with many automorphisms, https://arxiv.org/abs/1410.7031

[2] O. Ahmadi and G. McGuire, Curves over finite fields and linear recurring sequences, Surveys in Combinatorics 2015, Cambridge.

[3] O. Ahmadi, G. McGuire and A. Rojas-León, Decomposing Jacobians of curves over finite fields in the absence of algebraic structure, J. Number Theory 156 (2015), 414-431. MR3360347

[4] I. Blanco-Chacón, R. Chapman, S. Fordham, and G. McGuire (2016) Divisibility of Lpolynomials for a family of curves. Contemporary Developments in Finite Fields and Applications: pp. 1-10.

[5] R. Lidl, H. Niederreiter, Finite Fields, Addison-Wesley, 1983.

[6] G. McGuire, E. S. Y1lmaz, On the Zeta Functions of Supersingular Curves, preprint. 